$05,04,12$

\title{
Влияние орбитального упорядочения на спектры магнитного резонанса в зарядово-упорядоченных и фрустрированных манганитах
}

\section{() Л.Э. Гончарь}

Уральский государственный университет путей сообщения, Екатеринбург, Россия

Уральский федеральный университет им. Б.Н. Ельцина,

Екатеринбург, Россия

E-mail: I.e.gonchar@urfu.ru

\begin{abstract}
Проведено теоретическое исследование взаимосвязи кристаллической структуры, зарядовой, орбитальной и магнитной подсистем в зарядово-упорядоченных манганитах $R_{1-x} A_{x} \mathrm{MnO}_{3}$ (где $R^{3+}$ - редкоземельный ион, $A^{2+}$ - щелочноземельный ион, $\left.x=0.5,2 / 3\right)$. Использована модель орбитально-зависимых обменных взаимодействий и одноионной анизотропии. Показано наличие квази-низкоразмерных магнитных структур. Рассчитаны спектры спиновых волн и антиферромагнитного резонанса.
\end{abstract}

Работа выполнена при финансовой поддержке Правительства Российской Федерации, постановление № 211, контракт № 02.A03.21.0006.

DOI: 10.21883/FTT.2019.05.47577.24F

\section{1. Введение}

Кристаллы манганитов $R_{1-x} A_{x} \mathrm{MnO}_{3}$ (где $R^{3+}-$ редкоземельный ион, $A^{2+}$ - щелочноземельный ион, $x-$ степень допирования), являясь соединениями, в которых обнаружено колоссальное магнетосопротивление (КМС), обладают широким рядом свойств, интересных для изучения. Это структурные и магнитные фазовые переходы, особенности электрических и упругих свойств, анизотропия в различных подсистемах кристаллов [1]. Особая роль в исследованиях манганитов принадлежит как родительскому соединению $\mathrm{LaMnO}_{3}$, так и зарядовоупорядоченным фазам (ЗУФ) соединений с неизовалентным замещением редкоземельного элемента щелочноземельным. При определенных значениях $(x=1 / 4$, $1 / 2,2 / 3,3 / 4)$ носители заряда, образовавшиеся вследствие допирования, локализуются на ионах марганца и формируют упорядоченную структуру $\mathrm{Mn}^{3+} / \mathrm{Mn}^{4+}$ [2]. Орбитальная и магнитная структуры в этом случае значительно усложняются [3], возникают большие магнитные ячейки и фрустрированные структуры [4,5]. Магнитная структура сильно взаимосвязана с кристаллической, орбитальной и зарядовой структурами [2]. Спектры магнитных возбуждений могли бы уточнить степень влияния, однако большое количество магнитных взаимодействий существенно усложняет спектры [6]. Ранее в работе [7] было проведено измерение только небольшого фрагмента нижних ветвей спектра актиферромагнитного резонанса (АФМР). Другие измерения касались спектра спиновых волн соединений с отсутствующей локализацией заряда [8]. В работе [9] спектры спиновых волн для $\operatorname{Pr}_{0.5} \mathrm{Ca}_{0.5} \mathrm{MnO}_{3}$ измерены подробно вплоть до высоких энергий. Наличие таких экспериментов приводит к необходимости пересмотра модели $[6,10]$ или ее параметров.
При этом необходимо выяснить роль орбитальной и зарядовой структуры в формировании магнитной структуры и спектров магнитных возбуждений.

\section{2. Модель орбитальной структуры}

Кристаллическая структура зарядово-упорядоченных манганитов имеет низкую симметрию, в частности, в соединениях с $x=1 / 2$ она обладает моноклинной симметрией $P 2_{1} / m$ [4], а в соединениях с $x=2 / 3$ симметрия кристаллической структуры орторомбическая Рnma, но c утроенной ячейкой [5]. Особенно важной частью кристаллической структуры для описания орбитального упорядочения является зарядовый порядок, то есть способы чередования ионов $\mathrm{Mn}^{3+} / \mathrm{Mn}^{4+}$.

Трехвалентные ионы марганца в октаэдрическом кислородном окружении находятся в основном состоянии, описываемом мультиплетом ${ }^{5} E$ с орбитальными волновыми функциями $|\theta\rangle,|\varepsilon\rangle$. Эффективный гамильтониан, состоящий из линейной комбинации орбитальных операторов $e$-типа для каждого иона $\mathrm{Mn}^{3+}$ с номером $(i)$ может быть записан как [3]:

$$
H_{\mathrm{eff}}^{i}=\alpha_{1}^{i} X_{\theta}+\alpha_{2}^{i} X_{\varepsilon},
$$

где операторы в матричной форме имеют вид

$$
X_{\theta}=\left(\begin{array}{rr}
-1 & 0 \\
0 & 1
\end{array}\right) ; \quad X_{\varepsilon}=\left(\begin{array}{ll}
0 & 1 \\
1 & 0
\end{array}\right) .
$$

Модель позволяет менять только два параметра $\alpha_{1}^{i}, \alpha_{2}^{i}$ для каждого иона марганца. Эти параметры состоят из основного вклада - линейного вибронного взаимодействия, и дополнительных вкладов, таких как дальнее кулоновское взаимодействие или нелинейные вибронные взаимодействия. Орбитальная структура может 

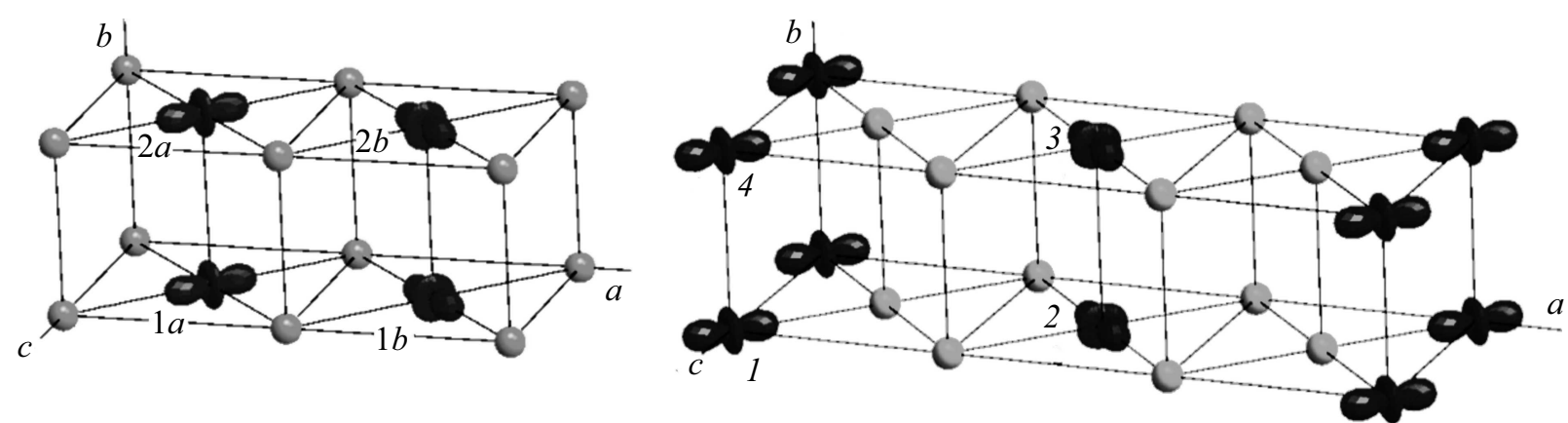

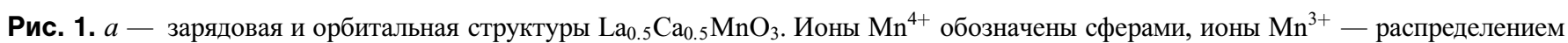
одноэлектронных плотностей. $b$ - зарядовая и орбитальная структуры $\mathrm{La}_{1 / 3} \mathrm{Ca}_{2 / 3} \mathrm{MnO}_{3}$. Ионы $\mathrm{Mn}^{4+}$ обозначены сферами, ионы $\mathrm{Mn}^{3+}$ - распределением одноэлектронных плотностей. В поле рисунка обозначены позиции $\mathrm{Mn}^{3+}$.

отличаться от структуры, обусловленной искажениями ближайшего окружения [3]. Для иона $\mathrm{Mn}^{3+}$ с номером $i$ многоэлектронная волновая функция основного состояния имеет вид

$$
\Psi_{i}=\left\{\begin{array}{l}
\left|\cos \frac{\Theta_{i}}{2}\right||\theta\rangle_{i}-\left|\sin \frac{\Theta_{i}}{2}\right||\varepsilon\rangle_{i}, \alpha_{2}^{i}>0, \\
\left|\cos \frac{\Theta_{i}}{2}\right||\theta\rangle_{i}+\left|\sin \frac{\Theta_{i}}{2}\right||\varepsilon\rangle_{i}, \alpha_{2}^{i}<0 .
\end{array}\right.
$$

Основная характеристика орбитального состояния $\mathrm{Mn}^{3+}$ - угол смешивания орбитальных функций $\Theta_{i}-$ зависит как от искажений локального кислородного окружения, так и от нелокальных и нелинейных факторов [11].

Зарядовое и орбитальное упорядочения в предлагаемой модели являются основой для описания магнитного упорядочения. Зарядовое и орбитальное упорядочения рассматриваемых кристаллов изображены на рис. 1.

\section{3. Модель магнитной структуры}

Гамильтониан магнитных взаимодействий, описывающих магнитную структуру соединений диэлектрических манганитов, состоит из трех орбитально-зависимых вкладов [6]:

$$
\begin{aligned}
\hat{H}_{m a g}= & \sum_{i>j} J_{i j}\left(\Theta_{i}, \Theta_{j}\right)\left(\mathbf{S}_{i} \mathbf{S}_{j}\right)+\sum_{i, \alpha, \beta} D_{i}^{\alpha, \beta}\left(\Theta_{i}\right) S_{i}^{\alpha} S_{i}^{\beta} \\
& +\mu_{\mathrm{B}} \sum_{i, \alpha, \beta} H_{\alpha} \mathbf{g}_{\alpha \beta}^{i}\left(\Theta_{i}\right) S_{i}^{\beta},
\end{aligned}
$$

где первое слагаемое отвечает за обменное взаимодействие, второе является энергией одноионной анизотропии, а третье описывает зеемановское взаимодействие с внешним магнитным полем; $\alpha, \beta=x, y, z, \mathbf{S}_{i}-$ полный спин магнитного иона, $\mathbf{H}$ - напряженность внешнего магнитного поля, $g_{\alpha \beta}^{i}$ - компоненты $g$-тензора.

В нашей модели в качестве обменного взаимодействия рассматривается сверхобменное взаимодействие между ближайшими магнитными соседями через про-

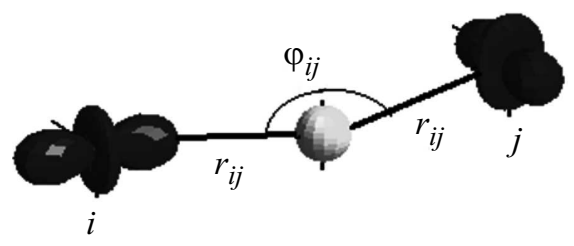

Рис. 2. Конфигурация сверхобменной связи $\mathrm{Mn}^{3+}-\mathrm{O}-\mathrm{Mn}^{3+}$. Ион кислорода обозначен сферой, ионы марганца - распределением электронных плотностей.

межуточный лиганд - ион $\mathrm{O}^{2-}$ (конфигурацию связи см. на рис. 2). Параметры сверхбменного взаимодействия зависят как от конфигурации сверхобменной связи, так и от орбитальных состояний взаимодействующих ионов марганца $[3,6,10]$ :

$$
\begin{aligned}
J_{i j}^{b}= & \frac{J_{0}^{(1)} \cos ^{2} \varphi_{i j}}{r_{i j}^{10}}\left(1-\alpha\left(\cos \Theta_{i}+\cos \Theta_{j}\right)\right. \\
& \left.+\beta \cos \Theta_{i} \cos \Theta_{j}\right)
\end{aligned}
$$

- взаимодействие между трехвалентными ионами марганца вдоль оси $b$,

$$
J_{i j}^{a c}=\frac{J_{0}^{(2)} \cos ^{2} \varphi_{i j}}{r_{i j}^{10}}\left(1+\alpha^{\prime} / 2\left(\cos \Theta_{i} \pm \sqrt{3} \sin \Theta_{i}\right)\right)
$$

- взаимодействие между ионами $\mathrm{Mn}^{3+}$ и $\mathrm{Mn}^{4+}$ в плоскости $a c$,

$$
J_{i j}^{b, a c}=\frac{J_{0}^{(3)} \cos ^{2} \varphi_{i j}}{r_{i j}^{10}}
$$

- взаимодействие между четырехвалентными ионами марганца, не обладающее орбитальной зависимостью.

Параметры зависимостей (5)-(7) определены в работах $[6,12]$ и составляют

$$
\begin{gathered}
J_{0}^{(1)}=1.24 \cdot 10^{3} \mathrm{meV} \cdot \AA^{10}, \quad \alpha=1.0, \quad \beta=4.5 \\
J_{0}^{(2)}=-4.22 \cdot 10^{2} \mathrm{meV} \cdot \AA^{10}, \quad \alpha^{\prime}=5.1 \\
J_{0}^{(3)}=0.97 \cdot 10^{3} \mathrm{meV} \cdot \AA^{10}
\end{gathered}
$$


Сравнение экспериментальных параметров сверхобменного взаимодействия с теоретическим расчетом

\begin{tabular}{c|l|l}
\hline Связь & $J, \mathrm{meV}-$ эксперимент & $\begin{array}{c}J, \text { meV - теория }[6,10,13] \\
\text { (диапазон по ряду 3У соединений })\end{array}$ \\
\hline $\mathrm{Mn}^{3+}-\mathrm{Mn}^{3+}(b)$ & $3.22[15] ; 2.09[9]$ & $1.55-2.67$ \\
\hline $\mathrm{Mn}^{3+}-\mathrm{Mn}^{4+}(a c)$ & $-6.76,0.75[15]$ & $-1.81 \div-2.5,0.58-1.03$ \\
& $-11.4,1.35[16]$ & \\
& $-9.98,1.83[14]$ & \\
\hline $\mathrm{Mn}^{4+}-\mathrm{Mn}^{4+}(b)$ & $3.22[15] ; 2.09[9]$ & $1.2-1.3$ \\
\hline $\mathrm{Mn}^{4+}-\mathrm{Mn}^{4+}(a c)$ & & 1.03
\end{tabular}

Параметры сверхобменных связей для рассматриваемых соединений различаются незначительно и примерно составляют $r \sim 1.95 \AA, \varphi \sim 155^{\circ}[4-6,10]$. Для связей в плоскости $a c$, где расстояния $\mathrm{Mn}-\mathrm{O}$ различны в силу кооперативного эффекта Яна-Теллера, в качестве $r$ берется средняя величина. Значение угла $\Theta$ в рамках данной работы можно принять равным $\sim 120^{\circ}$.

Обменные взаимодействия, полученные в этой модели, хорошо описывают магнитные структуры и температуру Нееля для ЗУ манганитов $(x=1 / 2,2 / 3) \quad[6,10,12,13]$ в модели среднего поля. Однако в дальнейшем будет показано, что параметры (6), относящиеся к сверхобменному взаимодействию $\mathrm{Mn}^{3+}-\mathrm{Mn}^{4+}$, нуждаются в пересмотре.

Одноионная анизотропия также учитывает орбитальное состояние [6]. Для каждого иона $\mathrm{Mn}^{3+}$ в локальных осях кислородного октаэдра она будет иметь вид

$$
H_{a n}^{(i)}=D_{i} S_{i z_{l}}^{2}+E_{n}\left(S_{i x_{l}}^{2}-S_{i y_{l}}^{2}\right) .
$$

где

$$
D_{i}=-3 P \cos \Theta_{i}, \quad E_{i}=-\sqrt{3} P \sin \Theta_{i},
$$

Значения $D_{n}$ оказываются положительными и одинаковыми для всех магнитных ионов в ячейке, а $E_{n}$ обладает разным знаком для различных ионов $\mathrm{Mn}^{3+}$. В случае учета симметрии в кристалле с $x=1 / 2$ величины констант для позиций $a$ и $b$ также будут различаться.

Величина $P$ определена в работе [6]: $P \approx-0.1 \mathrm{meV}$.

Свойства симметрии кристаллов манганитов определяются не только искажениями октаэдров янтеллеровского типа, но и поворотами [6]. В этом случае выражение для магнитной одноионной анизотропии следует привести к общей системе координат, при этом появятся недиагональные по спину компоненты. Это преобразование приводит к появлению легкой оси намагничивания в манганитах с $x=1 / 2$.

Учет взаимодействия с магнитным полем для трехвалентых ионов марганца также подразумевает зависимость от орбитального состояния [6]. В слабых магнитных полях этой зависимостью можно пренебречь, однако она может иметь значение при исследовании орбитального упорядочения.
Зависимость $g$-тензора ионов $\mathrm{Mn}^{3+}$ в локальных осях кислородного окружения от угла смешивания орбитальных функций имеет вид

$$
\begin{aligned}
H_{z \text { eem }}^{(i)}= & g_{1} \mu_{B}\left(\mathbf{S}_{i} \mathbf{H}\right)-\frac{1}{2} g_{2} \mu_{B}\left[\left(3 S_{i z_{l}} H_{z_{l}}-\mathbf{S}_{i} \mathbf{H}\right) \cos \Theta\right. \\
& \left.+\sqrt{3}\left(S_{i x_{l}} H_{x_{l}}-S_{i y_{l}} H_{y_{l}}\right) \sin \Theta_{i}\right]
\end{aligned}
$$

где параметры $g_{1}=1.89, g_{2}=0.047$ найдены во втором порядке теории возмущений по спин-орбитальному взаимодействию. Выражение (13) также следует привести к общей системе координат. Для четырехвалентного иона марганца $g$-фактор изотропен, $g=2$.

\section{4. Результаты и обсуждение}

\section{1. Обменное взаимодействие между ионами марганца разной валентности}

При моделировании спектра спиновых волн в рамках модели (4)-(13) для $x=0.5$ было проведено сравнение с экспериментальными данными работы [9]. Было обнаружено, что при параметрах сверхобмена (8)-(10) форма дисперсионной зависимости приближена к экспериментальной, однако величины максимальных энергий спектра более чем в три раза меньше экспериментальных. При сравнении обменных параметров, полученных в работах [9,14-16], с параметрами нашей модели в работах [6,10-13] выяснилось, что существенное расхождение наблюдается в величинах $J^{a c}(9)$, то есть, во взаимодействии $\mathrm{Mn}^{3+}-\mathrm{Mn}^{4+}$. Сравнение приведено в таблице.

Учитывая особенности микроскопического описания обменных параметров в работе [12], можно предположить, что использование сверхобменных взаимодействий в оксидных соединениях, содержащих ионы меди и железа, не позволяет корректно описать обменные взаимодействия ионов марганца.

В связи с этим возникла необходимость пересмотра параметров (9) в формуле (7). Поскольку модель в работе [12] содержит большое количество вкладов, для ка- 


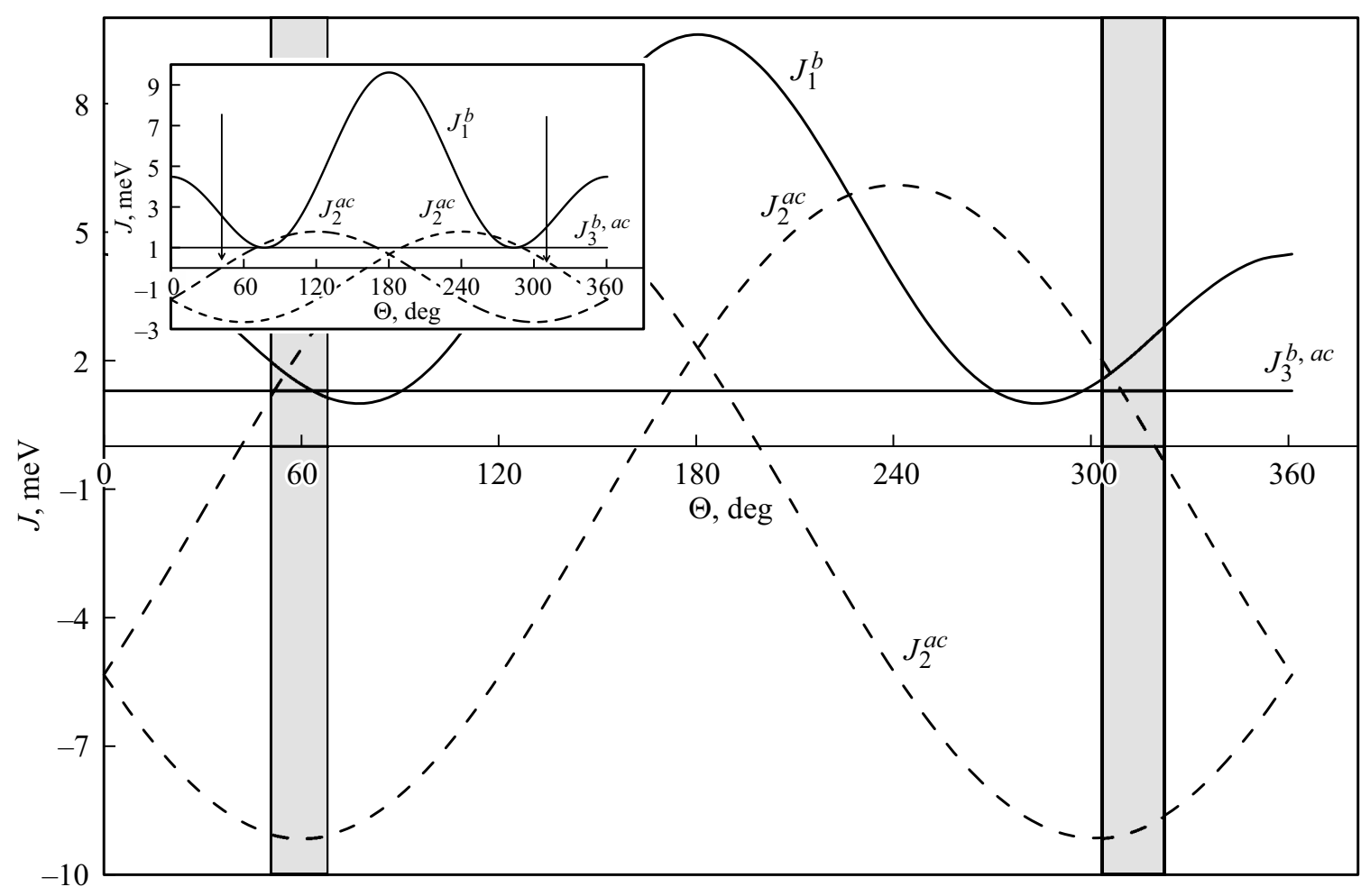

Рис. 3. Зависимость параметров сверхобменного взаимодействия от угла смешивания орбитальных функций, измененных в соответствии с экспериментальными данными [9]. На графике выделены области углов, дающих низкоразмерную структуру. На вставке - график зависимости сверхобменных параметров в соответствии с моделью [10-13].

чественной оценки обменных параметров $\mathrm{Mn}^{3+}-\mathrm{Mn}^{4+}$ достаточно внести изменения в феноменологические параметры:

$$
J_{0}^{(2)}=-2.2 \cdot 10^{3} \mathrm{meV} \cdot \AA^{10}, \quad \alpha^{\prime}=3.1 .
$$

При этом необходимо получить новый график зависимости сверхобмена от угла смешивания орбитальной структуры $\Theta$. Новый график представлен на рис. 3. Основные характеристики зависимости по-прежнему не противоречат правилам Гуденафа-Канамори-Андерсона [2] для определения знака и относительной величины взаимодействий ионов с орбитальным вырождением. Значения обменных параметров $J_{1}^{a c} \approx-9.5 \mathrm{meV}$, $J_{2}^{a c} \approx 1.3 \mathrm{meV}$ для $\Theta \sim 120^{\circ}$.

Увеличение обменных параметров $\mathrm{Mn}^{3+}-\mathrm{Mn}^{4+}$ существенно увеличивает значение температуры Нееля в модели среднего поля. Таким образом, возникает противоречие: прежние значения хорошо описывают температуру Нееля, а скорректированные величины спектры спиновых волн. Эта проблема решается в рамках представлений о низкоразмерных и близких к ним структурах. В той части орбитальной структуры, которая наблюдается экспериментально, соотношение обменных параметров таково, что ферромагнитный обмен, связывающий магнитный зигзаг, значительно сильнее, чем антиферромагнитный обмен между зигзагами. С учетом данного соотношения можно рассматривать магнитную подсистему соединений с $x=1 / 2$ и $x=2 / 3$ как низкоразмерную. Для $x=1 / 2$ это квазиодномерные ферромагнитные „зигзаги“, изображенные на рис. 4, $a$. Для $x=2 / 3$ это сильно связанные тримеры, объединенные в полосы (см. рис. 4, $b$ ). Обе структуры не претерпели существенных изменений по сравнению с экспериментальными [4,5] и теоретическими [3].

Одноионная анизотропия подрешетки трехвалентных ионов марганца в соответствии с (11), (12) выделяет на каждом ионе легкую магнитную ось, параллельную одной из псевдоперовскитных осей и совпадающую с направлением связи $\mathrm{Mn}^{3+}-\mathrm{Mn}^{4+}$. Наличие поворотных искажений кислородного окружения $\mathrm{Mn}^{3+}$ и промежуточные ионы $\mathrm{Mn}^{4+}$ без одноионной анизотропии, а также ферромагнитный обмен внутри „зигзага“, приводят магнитную структуру манганита с $x=1 / 2$ к одноосной с направлением легкой оси примерно вдоль псевдоперовскитной диагонали. В случае $x=2 / 3$ между ионами $\mathrm{Mn}^{3+}$ располагаются два иона $\mathrm{Mn}^{4+}$, антиферроманитные связи между которыми фрустрированы [3,5]. В связи с этим, независимо от поворотных искажений октаэдрического окружения, в данном соединении представлена двухосная магнитная структура. Фрагменты с одинаковым направлением легкой оси ориентированы вдоль оси $c$ и образуют страйпы (рис. $4, b$ ).

В работах [9,14-16] в качестве дополнения к сверхобменному взаимодействию между ближайшими магнит- 

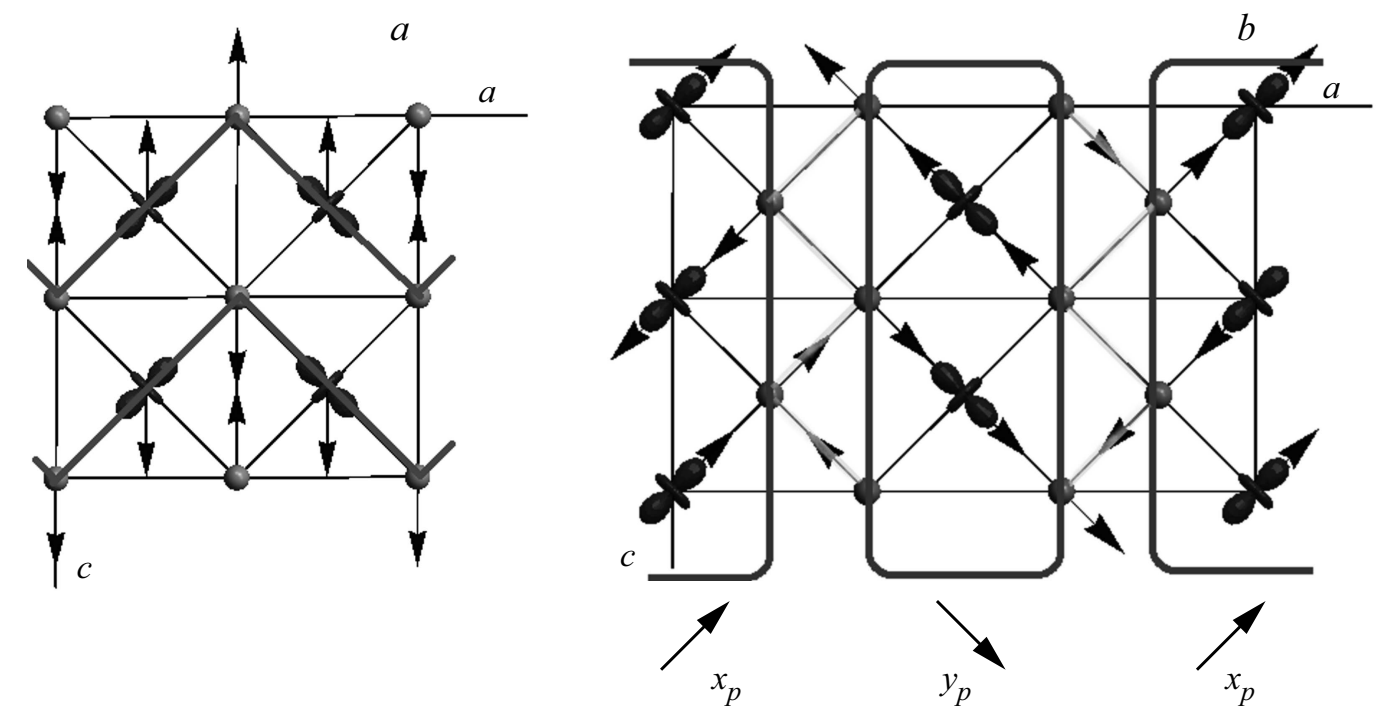

Рис. 4. $a-$ орбитальная и магнитная ( $C E$-тип) структуры $R_{0.5} \mathrm{Ca}_{0.5} \mathrm{MnO}_{3}$ в плоскости ас, жирной линией выделены ферромагнитные „зигзаги“; $b$ - орбитальная и магнитная структуры $R_{1 / 3} \mathrm{Ca}_{2 / 3} \mathrm{MnO}_{3}$ в плоскости $a c$, бледной линией выделены фрустрированные связи, прямоугольником обведены страйпы без фрустрированных связей; направления тримеров совпадают с псевдоперовскитными осями и отмечены жирными стрелками под рисунком. Сферы обозначают ионы Мn ${ }^{4+}$, распределения электронных плотностей $-\mathrm{Mn}^{3+}$, тонкие стрелки - направления магнитных моментов. Магнитные моменты в соседних плоскостях имеют противоположные направления.

ными соседями введены параметры обменных взаимодействий для вторых и третьих соседей. Эти параметры необходимы авторам указанных работ для улучшения количественного совпадения спектров с экспериментом. Однако микроскопические причины появления этих взаимодействий пока не представлены. В настоящей работе исследуется качественное влияние орбитальной структуры на спектры возбуждений, поэтому параметры обменного взаимодействия с дальними соседями в расчетах не учитываются.

\section{5. Спектры магнитных возбуждений}

Для расчета дисперсионных зависимостей спиновых волн в ЗУ соединениях был использован гамильтониан (4) с учетом обменных взаимодействий (5)-(7) с параметрами (8), (10), (14). Зависимости рассчитаны в линейном приближении. Для сравнения с экспериментом [9] в соединении манганита с $x=1 / 2$ было рассмотрено направление $[h 00]$ вдоль ,зигзага“. Результаты моделирования представлены на рис. 5. Характерные особенности экспериментального спектра [9] качественно воспроизводятся в данной модели. Хорошо заметно при сравнении с теоретическими спектрами, полученными с использованием модели (4)-(13) [6], что вблизи Г-точки нижние ветви, ответственные за спектр АФМР, не изменили своего положения, поскольку связаны с одноионной анизотропией и межплоскостным обменным взаимодействием.

Полевые и угловые зависимости спектра АФМР для $3 У$ манганита $\operatorname{Pr}_{0.5} \mathrm{Ca}_{0.5} \mathrm{MnO}_{3}$ представлены на рис. 6 .
Низкополевая зависимость спектра АФМР в плоскости $a c$ (рис. $6, b)$ отражает наличие ярко выраженной легкой оси, тогда как характер зависимости при полях выше критического демонстрирует двухосное поведение, связанное с орбитальной структурой (рис. 6,c). Таким образом, упрощенные модели типа $D S_{i z}^{2}$ не могут быть использованы для манганитов.

Полевая зависимость нижних частот спектра АФМР в направлении легкой оси при изменении модели расчета $J$ (рис. 6, $a$ ) практически не претерпела изменений по сравнению с предыдущей работой [6] и хорошо согласуется с экспериментом [7] (рис. 6,d). Угловая зависимость (рис. 6,c) приобрела менее выраженный характер. Расчет спектров спиновых волн и АФМР для ЗУ манганитов с другим редкоземельным ионом качественно не отличается от рис. 6, однако, отсутствуют экспериментальные данные для сравнения. Небольшие изменения угла смешивания орбитальной структуры $\Theta$ не сказываются на зависимостях существенным образом.

Моделирование для $x=2 / 3$ было проведено в рамках тех же моделей. Изменения в модельных величинах обменного взаимодействия в плоскости $a c$ не влекут за собой уменьшения или снятия конкуренции в магнитных взаимодействиях. Как видно из рис. 3, для характерных углов смешивания орбитальной структуры (затененная область) антиферромагнитные обменные взаимодействия внутри плоскости близки по величине.

Дисперсии спиновых волн при условии магнитной фрустрации имеют сложный характер. Для манганита $\mathrm{La}_{0.5} \mathrm{Ca}_{0.5} \mathrm{MnO}_{3}$ это 24 ветви, образующие шесть зон с энергиями от 3 до $70 \mathrm{meV}$. В самой нижней зоне 


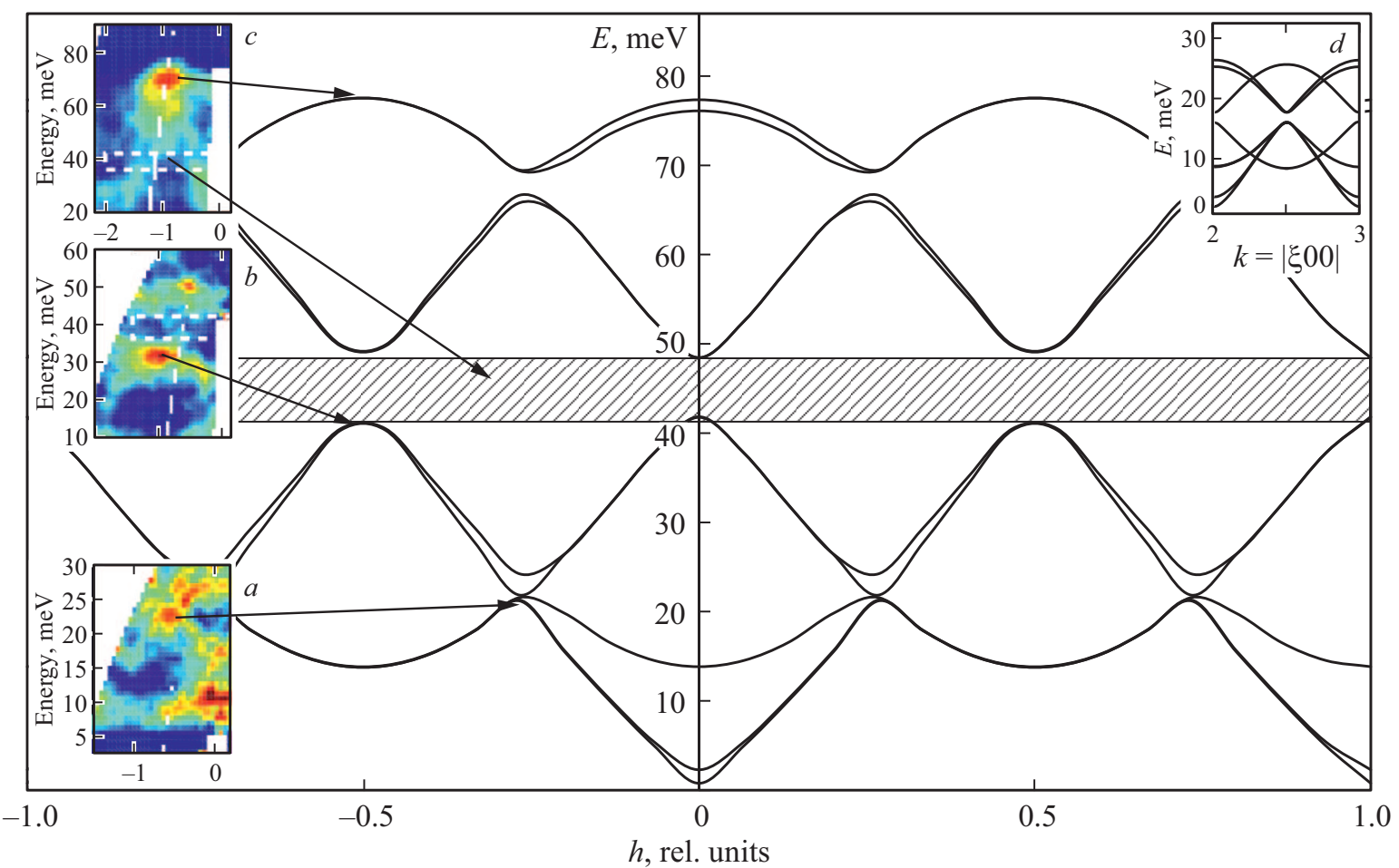

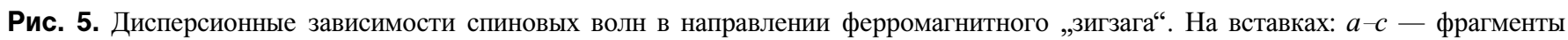
экспериментальных спектров из работы [9], $d$ - результат моделирования в предыдущей работе [6]. Стрелками отмечены характерные особенности экспериментального спектра, присутствующие в теоретической зависимости.
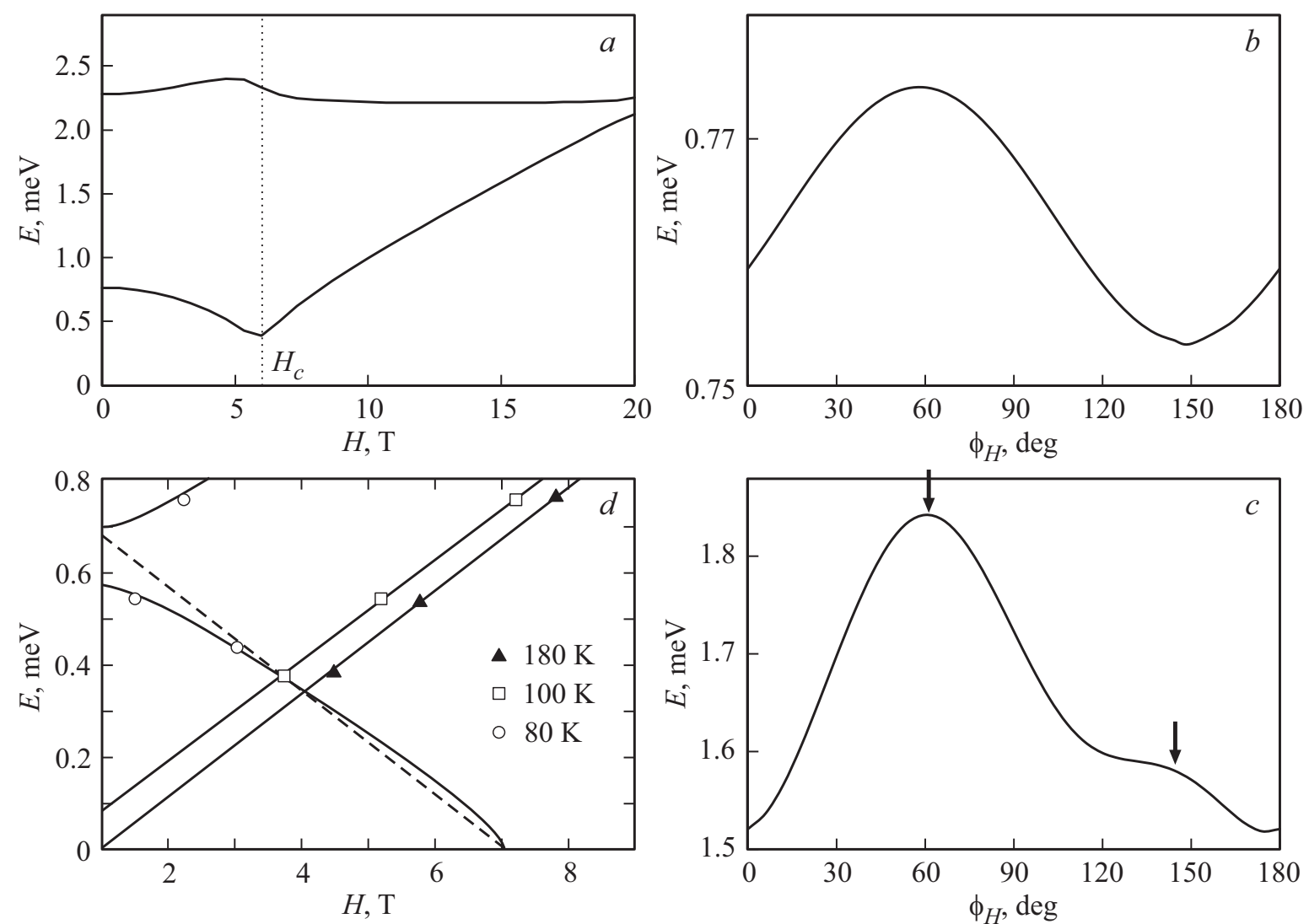

Рис. 6. Спектры АФМР для $\operatorname{Pr}_{0.5} \mathrm{Ca}_{0.5} \mathrm{MnO}_{3}$. $a-$ расчетная полевая зависимость спектра АФМР нижних ветвей; $b, c-$ расчетные угловые зависимости для $H=10$ и $H=150 \mathrm{~T}$ соответственно (направление внешнего поля меняется в плоскости $a c$, угол отсчитывается от направления связи $\left.\mathrm{Mn}^{3+}-\mathrm{Mn}^{4+}\right) ; d-$ экспериментальная полевая зависимость спектра АФМР для $\operatorname{Pr}_{0.5} \mathrm{Ca}_{0.5} \mathrm{MnO}_{3-\delta}[7]$. 


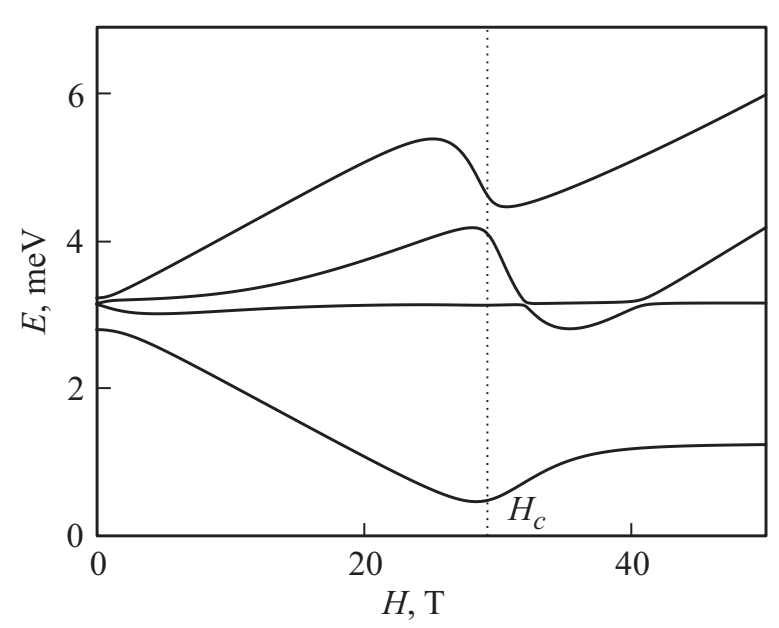

Рис. 7. Расчетная полевая зависимость спектра АФМР нижних ветвей для $\mathrm{La}_{1 / 3} \mathrm{Ca}_{2 / 3} \mathrm{MnO}_{3}$ в направлении внешнего магнитного поля вдоль тримеров (рис. $4, a$ ).

содержатся четыре ветви спиновых волн. Полевая зависимость нижних ветвей спектра АФМР соответствует двухосной макнитной структуре и имеет одинаковый характер вдоль обеих осей тримеров. Она характеризуется довольно высоким критическим полем (рис. 7).

\section{6. Заключение}

Рассмотрены зарядово-упорядоченные соединения псевдоперовскитных манганитов со степенью допирования $x=1 / 2$ и $2 / 3$. Выполнена модификация модели орбитально-зависимого обмена с учетом экспериментальных дисперсионных зависимостей [9]. Показано, что благодаря зарядово-орбитальной структуре в ЗУ фазе манганитов формируются квази-низкоразмерные магнитные структуры. Рассчитаны полевые зависимости спектров АФМР. Показано, что учет орбитально-зависимой одноионной анизотропии формирует двухосную магнитную структуру. Зарядово-орбитальное упорядочение качественно формирует спектры АФМР, однако уточнить угол смешивания орбитальных функций по резонансным спектрам невозможно.

\section{Список литературы}

[1] Н.Г. Бебенин, Р.И. Зайнуллина, В.В. Устинов. УФН 188, 801 (2018).

[2] J.B. Goodenough. Phys. Rev. 100, 564 (1955); E.O. Wollan, W.C. Koehler. Phys. Rev. 100, 545 (1955).

[3] L.E. Gonchar. J. Magn. Magn. Mater. 465, 661(2018).

[4] P.G. Radaelli, D.E. Cox, M. Marezio, S.-W. Cheong. Phys. Rev. B 55, 3015 (1997).

[5] P.G. Radaelli, D.E. Cox, L. Capogna, S.-W. Cheong, M. Marezio. Phys. Rev. B 59, 14440 (1999).

[6] Л.Э. Гончарь, А.Е. Никифоров. ЖЭТФ 123, 575 (2003).

[7] S. Kawamata, S. Noguchi, K. Okuda, H. Nojiri, M. Motokawa. J. Magn. Magn. Mater. 226-230, 854 (2001).
[8] H.Y. Hwang, P. Dai, S.-W. Cheong, G. Aeppli, D.A. Tennant, H.A. Mook. Phys. Rev. Lett. 80, 1316 (1998).

[9] R.A. Ewings, T.G. Perring, O. Sikora, D.L. Abernathy, Y. Tomioka, Y. Tokura. Phys. Rev. B 94, 014405 (2016).

[10] L.E. Goncthar, A.E. Nikiforov. Phys. Rev. B 66, 014437 (2002).

[11] L.E. Goncthar, A.E. Nikiforov. Phys. Rev. B 88, 094401 (2013).

[12] L.E. Gontchar, A.E. Nikiforov, S.E. Popov. J. Magn. Magn. Mater. 223, 175 (2001).

[13] L.E. Gontchar, A.E. Nikiforov, S.E. Popov. J. Magn. Magn. Mater. 300, 167 (2006).

[14] D. Senff, F. Krüger, S. Scheidl, M. Benomar, Y. Sidis, F. Demmel, M. Braden. Phys. Rev. Lett. 96, 257201 (2006).

[15] H. Ulbrich, F. Krüger, A.A. Nugroho, D. Lamago, Y. Sidis, M. Braden. Phys. Rev. B 84, 094453 (2011).

[16] G.E. Johnstone, T.G. Perring, O. Sikora, D. Prabhakaran, A.T. Boothroyd. Phys. Rev. Lett. 109, 237202 (2012).

Редактор Е.Ю. Флегонтова 